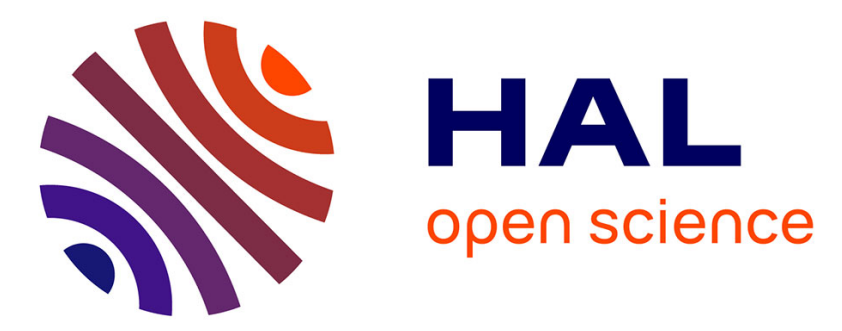

\title{
A fuzzy reasoning classification method for pattern recognition
}

Emmanuel Schmitt, Cyril Mazaud, Vincent Bombardier, Pascal Lhoste

\section{To cite this version:}

Emmanuel Schmitt, Cyril Mazaud, Vincent Bombardier, Pascal Lhoste. A fuzzy reasoning classification method for pattern recognition. 15th International Conference on Fuzzy Systems, FUZZIEEE'06, Jul 2006, Vancouver, Canada. pp.5998-6005. hal-00087474

\section{HAL Id: hal-00087474 https://hal.science/hal-00087474}

Submitted on 2 Oct 2006

HAL is a multi-disciplinary open access archive for the deposit and dissemination of scientific research documents, whether they are published or not. The documents may come from teaching and research institutions in France or abroad, or from public or private research centers.
L'archive ouverte pluridisciplinaire HAL, est destinée au dépôt et à la diffusion de documents scientifiques de niveau recherche, publiés ou non, émanant des établissements d'enseignement et de recherche français ou étrangers, des laboratoires publics ou privés. 


\title{
A Fuzzy Reasoning Classification Method for Pattern Recognition
}

\author{
Emmanuel Schmitt, Cyril Mazaud, Vincent Bombardier, and Pascal Lhoste
}

\begin{abstract}
This paper focuses on a Fuzzy Reasoning Classification Method to improve the potential of pattern recognition in the automated inspection and classification of wooden boards. After the definition of the characteristic features, we implement a fuzzy inference mechanism allowing to take into account the subjectivity of the human visual system. In this article, we have decided to work on the distribution and the representation of the Information. In this sense, our study speaks about the impact of fuzzification on the recognition rates and the structure of our decision module. The part concerning the classification mechanism allows ourselves to integrate knowledge in the generation of the numeric model. This knowledge is enquired at the different field experts thanks to the NIAM formalism. The results, which are presented on a generic benchmark and real data, show the efficiency of such an approach.
\end{abstract}

\section{INTRODUCTION}

$\mathrm{T}$ HE aim of the paper herein is to present a novel approach of pattern recognition in the field of industrial vision system and more precisely, the system used in the wood product industry. In fact, the furniture suppliers want to improve the wooden products quality which is essentially defined by the lack of defects and the color uniformity. In general, the defect definition and the color grading procedure are highly subjective and require human intervention. Thus the decision on the wooden boards sorting is linked to the different human operators understanding. That's why, to develop a reliable vision system, we need more and more Information on the acquired images to obtain a better control of the vision system and of the processes in relation with it (sorting, cutting). To study the images which are provided by the vision system, we can consider another source of Information from human expert Knowledge. These data concern rather qualitative Information. To summarize, the global framework of this

This work was supported in part by the Luxembourger Ministry of Culture, Higher Education and Research under Grant BFR and the LuxScan Technologies Company.

F. E. Schmitt is with the Automatic Research Center of Nancy (e-mail emmanuel.schmitt@cran.uhp-nancy.fr) and the Luxscan Technologies company - ZARE Ouest - L-4384 EHLERANGE, Luxembourg.

S. C. Mazaud is with the Automatic Research Centre of Nancy (e-mail: cyril.mazaud@cran.uhp-nancy.fr) and the Luxscan Technologies company.

T. V. Bombardier is with the Automatic Research Centre of Nancy CRAN, CNRS, UMR 7039 - CRAN - Faculté des Sciences - Bd des Aiguillettes - BP 239, 54506 VANDOEUVRE-LES-NANCY Cedex, France (phone: +33-3-83-68-44-52; fax: +33-3-83-68-44-59; e-mail: vincent.bombardier@cran.uhp-nancy.fr).

F. P. Lhoste is with Research dEpartment in Innovative Processes ERPI, EA 3767, ENSGSI - INPL - 8 rue Bastien Lepage - BP 647, 54010 NANCY Cedex, France (phone: +33-3-83-19-32-45; fax: +33-3-83-19-3200; e-mail: pascal.lhoste@ensgsi.inpl-nancy.fr). project is to define a modelling and an integrating method, which allows, on the one hand, to transform Knowledge into Information, on the other hand, to extract Information from Numerical Data measured by the system and finally to integrate these Information in a same cognitive Information referential system.

There are lots of methods to identify and classify the patterns observed on the acquired images: statistical algorithms, neural network, fuzzy logic classifiers ... But in order to ensure a real-time recognition adapted to our subjective problem, we have decided to base our developments on the fuzzy logic. In this sense, we present here a method using Fuzzy Inference System based on linguistic rules. After a short presentation of the framework, we explain our methodology of classification by stressing the impact of the features fuzzification. Then, we expose how the knowledge integration into our classifier structure can improve its quality, in term of recognition rate.

\section{FRAMEWORK}

The works here presented take place in a francoluxembourger collaboration between the Automatic Research Center of Nancy (CRAN) and the LuxScan Technologies Company. They concern the development of a recognition vision system for defects identification and color classification on wooden boards. Defects and colors characterization defines Information, which are used to estimate the quality of the final products. This quality is determined on production lines in real time. Its speed can reach 3 to 5 meters per second with a maximum of 200 defects per meter. Fig. 1 illustrates the functioning principle of the vision system.

In order to improve the reliability, we use a vision system, which is made up of two kinds of linear sensors. We use them in a combined way or in an independent way to acquire

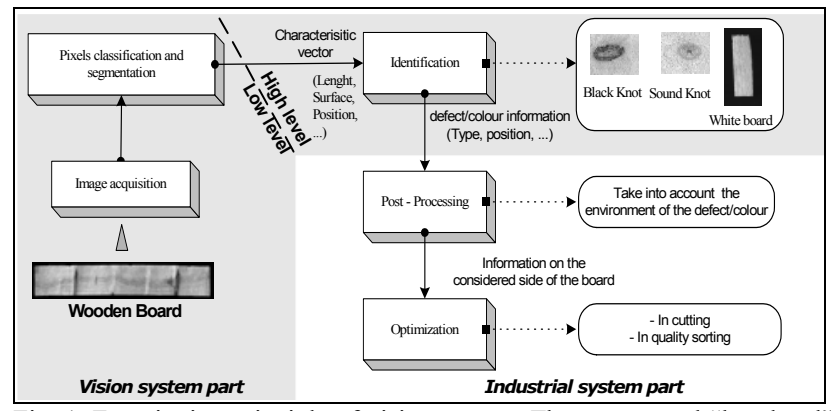

Fig. 1. Functioning principle of vision system. The part named "low level" deals with the acquisition of the wooden board images thanks to the different sensors and the first step of image processing (signals processing). The "high level" part concerns information processing from defect/color identification to board optimization. 
the images of wood. On the one hand, we use laser sources in order to define the density, the orientation of fibres, the red and infrared intensities. On the other hand, we need color cameras to identify the defect of coloration and the aspect of the boards. These sensors return three components: red, green and blue signals. Signals of all sensors are sampled at $2 \mathrm{kHz}$ and represented with a 256-level greyscale. During the image segmentation, a set of features (surface, color, orientation, ...) is calculated on the different regions to provide a characteristic vector used by the recognition step. The works that we present concern only the identification module situated in the high level of the industrial vision system illustrated by Fig. 1 .

\section{IDENTIFICATION METHODOLOGY}

\section{A. State of the art}

In the field of wooden board classification, several kinds of sensors are used: cameras, ultrasonic, X-ray [1]. Most of classifiers are based on supervised or unsupervised neural networks [2]-[4]. In spite of the results exposed in these papers, we want to bring a novel approach using tools from fuzzy sets [5] and more precisely from fuzzy logic [6]. This choice is easily justifiable with three ideas.

Initially, the defects and the colors that we classify are intrinsically fuzzy. By example, it doesn't exist a strict boundary between sound wood and defects. It is the same for the wood color. Actually, the features calculated on the images are precise but uncertain. Using fuzzy logic allows to minimize this effect. Secondly, the output classes of defects or colors are not disjointed, they are intrinsically fuzzy. In fact, it exists several shades for one wood color. By example, for a Red hue, we can detect three different shades: light red, medium red and dark red. Finally, the knowledge expressed by the experts is often uncertain (a color rather brown). That's why we represent this knowledge in the form of fuzzy terms. Moreover, this kind of language could improve the human comprehension of the system. We also remark that classical logic is not appropriated to represent the human visual system. It is too restrictive concerning the modelling of linguistic concepts.

We have decided to use a method based on the fuzzy set theory [5], [7] to reply to these criteria. This theory allows to keep the subjectivity notion in the taken decisions. Our vision system provides numerical data in the form of a characteristic vector. There are lots of methods using Approximate Reasoning on numerical data. In our case, fuzzy logic concept and especially fuzzy linguistic rules [8] are appropriated.

\section{B. Fuzzy reasoning}

It exits different kinds of rules allowing to define a fuzzy inferences engine: conjunctive rules and implicatives rules, which put together possibility and anti-gradual rules, and certitude and gradual rules. These rules are obtained from two ways: either by knowledge given by the expert or by learning from a data sample. Our inference mechanism activates in parallel a rules base $\kappa$. Each rules provides a partial conclusion, which has to be unified to give a final conclusion. Equation (1) defines the rules base.

$$
R^{\kappa}=\bigcup_{i=1, \ldots, n} A_{i} \times B_{i}
$$

The union operator must be a conjunction operator ( $T$ norm: $\mathrm{T})$ if we use implicative rules and a disjunction operator (T-Conorm: $\perp$ ) in the case of conjunctive rules [9].

Two reasoning using fuzzy rules are distinguished: deductive reasoning and abductive reasoning. In the first case, we want to find $\mathrm{Y}$ thanks to $\mathrm{X}$ values. In the second case, we want to obtain information about the input $\mathrm{X}$ thanks to knowledge about the output Y. In our case, we choose the deductive reasoning because it corresponds to the Modus Ponens. Moreover, we have chosen a conjunctive reasoning engine because our rules are firstly obtained from numerical data [8]; each rule is activated in parallel, and a disjunction operator combines intermediate results. So, we avoid redundancy in the rules base [9]. The two main models using these rules are Mamdani model and Larsen model [6].

\section{Fuzzy Reasoning Classifier (F.R.C.)}

Our method allows to obtain automatically fuzzy rules according to a data set. Our fuzzy inferences mechanism [11], [12] follows the Larsen model because the Product is more adapted than the Minimum for the use of several premises [10]. We use the iterative method [13], which supports the rule with the maximum response. To keep the results gradual, we don't need the boundary refining.

1) Principle: We implement a supervised classifier. Our mechanism generates 'IF... THEN..." fuzzy rules, which characterize the defects and the colors classes. The algorithm is decomposed into three parts: the features fuzzification, the fuzzy rules generation and the model adjustment. Then the obtained set of rules is used to process the identification of the "unknown" defects.

2) Fuzzification of the features: The fuzzification consists in parameters decomposition on several terms. Generally, the number of terms is empirically chosen, but this number has directly an impact on the recognition rates. Each part of this decomposition is expressed by a word of the natural language to make the link with the linguistic variables. By example, the color shade can be "light",

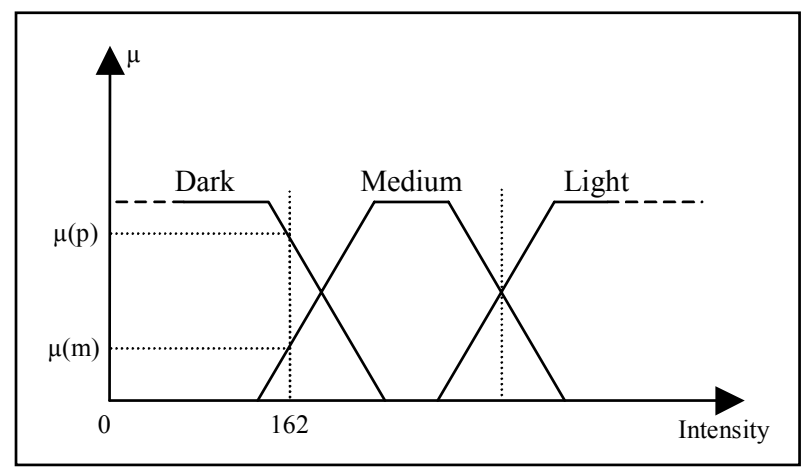

Fig. 2. Intensity level (shade) fuzzification in three terms. 
"medium", and "dark". From this decomposition, membership functions are described thanks to the analysis of samples set used for the numeric model generation. Fig. 2 gives an example of decomposition.

The graph illustrates one feature (intensity) represented by their three distributed terms on its definition field called universe of discourse. It is to be noted that the number of decomposition terms defines the rules like the term number, the form of the fuzzification curves has an impact on the global recognition rates. On Fig. 2, we have represented the decomposition following trapezial fuzzification. We have made some comparative tests about the fuzzification curves and the results are exposed later in this paper.

3) Linguistic rules generation: This step creates our numeric model. The algorithm automatically generates fuzzy rules like "IF... THEN...". This model describes how the system perceives the patterns. We use a training sample set to create the model. Generally speaking, if we consider two parameters in input $\left(\mathrm{V}_{\mathrm{i}}\right.$ and $\left.\mathrm{V}_{\mathrm{i}+1}\right)$ and one output $\left(\mathrm{Z}_{\mathrm{k}}\right)$, the fuzzy rule follows the form:

IF $V_{i}$ is $A_{j}$ AND IF $V_{i+1}$ is $A_{j+1}$

THEN $Z_{k}$ is in the defect class $C_{n}$

where $V_{i}$ and $V_{i+1}$ are the input data, $Z_{k}$ is the output data, $A_{j}$ and $A_{j+1}$ are the fuzzy subsets and $C_{n}$ is the class of $n^{\text {th }}$ pattern.

In this example, the "AND" operator corresponds to the Cartesian product between the two variables $V_{i}$ and $V_{i+1}$. This operation is done with the Product T-Norm (2).

$$
\mathrm{T}(\mathrm{x}, \mathrm{y})=\mathrm{x} * \mathrm{y}
$$

Then, the fuzzy inference engine is based on the use of Maximum/Product composition law. This inference follows Larsen's model [6], which uses a pseudo-implication operator represented by the Product. Finally, each rule returns a partial conclusion, which is aggregated with the others according to a disjunctive fuzzy operator. We use the maximum value according to Zadeh to realize this part (3).

$$
\perp(\mathrm{x}, \mathrm{y})=\max (\mathrm{x}, \mathrm{y})
$$

4) Model adjustment: F.R.C. has a model adjustment step [13]. This step is realized by the iterative part of the algorithm. In fact, it consists to set up the splitting of the representation space of the input attributes. This adjustment is made according to the results given by the training samples set. The process is the following:

- The algorithm generates a first model.

- If the classification rate is below a threshold $\sigma$ defined by the user, the iterative part readjusts this model.

The supervised training step must be realized with lots of attention by selecting representative samples of each defect and color classes. The general principle of the recognition module is illustrated with Fig. 3. the basic algorithm proposes an add-on refining step. This part allows to improve the membership degree of maximum membership class by modifying the slope of membership function. In our

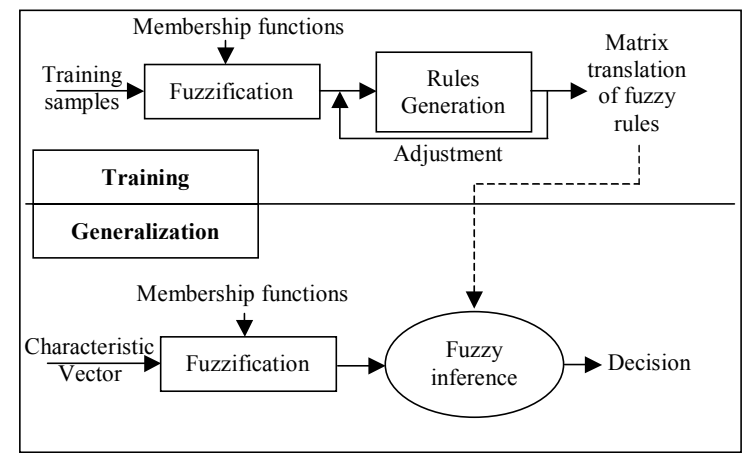

Fig. 3. Recognition module.

case, we want to keep the gradualities of the answers to avoid errors during the defects or colors identification.

\section{Impact of fuzzification on the recognition rate}

As said before, two fuzzification parameters may have an impact on the recognition rate. For that, we have realized some tests from several data sets: Iris benchmark, Color samples and Defect database. The Iris database is an international reference to characterize the potential of a classifier composed of 150 samples splitting in 3 output classes. The results exposed in Table 1 are obtained during the training step, i.e. 150 samples used for the model generation and the same ones for the recognition step. The Color database is compound of a 209-sample set for the models generation and a 1105-sample set for the generalization of our method. The Defect database is composed of 128 defects for the training part and 195 defects for the generalization part of the model. Notice that the Color and Defect databases are industrial samples taken from the industrial process. Few samples are used in training step because our method needs a small training sample (approximately fifteen samples for each class).

These tests have been made from a single fuzzy inferences engine with an equal distribution of the fuzzification terms. In this sense, we only use one decisionmaking node. We can notice that our methodology gives correct recognition rates in comparison to a bayesian classifier on Iris database (Table. I).

1) Influence of the fuzzification term number: The number of fuzzification terms has an influence on the rates. The best result is obtained for seven and nine fuzzification

TABLE I

\begin{tabular}{|c|c|c|c|c|c|}
\hline \multicolumn{5}{|c|}{ Fuzzy Method } & $\begin{array}{l}\text { Statistic } \\
\text { Method }\end{array}$ \\
\hline $\begin{array}{l}\mathrm{N}^{\circ} \text { of } \\
\text { terms }\end{array}$ & $\begin{array}{l}\text { Trapezial } \\
\text { curves }\end{array}$ & $\begin{array}{c}\text { Triangular } \\
\text { curves }\end{array}$ & $\begin{array}{c}\text { Gaussian } \\
\text { curves }\end{array}$ & $\begin{array}{c}\text { Trapezial + } \\
\text { Triangular } \\
\text { curves }\end{array}$ & $\begin{array}{l}\text { Bayesian } \\
\text { Classifier }\end{array}$ \\
\hline 2 & $92.0 \%$ & $92 \%$ & $88.0 \%$ & $92.0 \%$ & \multirow{6}{*}{$96.8 \%$} \\
\hline 3 & $96.7 \%$ & $96.7 \%$ & $92.0 \%$ & $96.7 \%$ & \\
\hline 5 & $96.7 \%$ & $96.7 \%$ & $96.7 \%$ & $96.7 \%$ & \\
\hline 7 & $98.0 \%$ & $98.0 \%$ & $98.0 \%$ & $98.7 \%$ & \\
\hline 9 & $100.0 \%$ & $100.0 \%$ & $98.0 \%$ & $100.0 \%$ & \\
\hline 11 & $96.0 \%$ & $96.0 \%$ & $96.0 \%$ & $96.0 \%$ & \\
\hline
\end{tabular}

RECOGNITION RATES ON IRIS BENCHMARK 
TABLE II

RECOGNITION RATES ON COLOR SAMPLES IN GENERALIZATION

\begin{tabular}{ccccc}
\hline \hline $\begin{array}{c}\text { Numbers of } \\
\text { fuzzification } \\
\text { terms }\end{array}$ & $\begin{array}{c}\text { Trapezial } \\
\text { curves }\end{array}$ & $\begin{array}{c}\text { Triangular } \\
\text { curves }\end{array}$ & $\begin{array}{c}\text { Gaussian } \\
\text { curves }\end{array}$ & $\begin{array}{c}\text { Trapezial }+ \\
\text { Triangular } \\
\text { curves }\end{array}$ \\
\hline 2 & $47.1 \%$ & $45.3 \%$ & $41.9 \%$ & $46.8 \%$ \\
\hline 3 & $80.9 \%$ & $79.7 \%$ & $56.2 \%$ & $80.1 \%$ \\
\hline $\mathbf{5}$ & $\mathbf{8 4 . 2 \%}$ & $\mathbf{8 4 . 7 \%}$ & $\mathbf{7 8 . 9 \%}$ & $\mathbf{8 2 . 1 \%}$ \\
\hline 7 & $83.8 \%$ & $83.2 \%$ & $78.1 \%$ & $79.5 \%$ \\
\hline 9 & $74.2 \%$ & $78.3 \%$ & $73.2 \%$ & $71.5 \%$ \\
\hline 11 & $73.9 \%$ & $71.2 \%$ & $72.9 \%$ & $70.6 \%$ \\
\hline \hline
\end{tabular}

terms, but for eleven terms, the over-fuzzification phenomenon appears implicating a degradation of the recognition rate. Thus, we must adapt the fuzzification to our problem. On real data, the general behavior is the same: the recognition rate increases with the number of fuzzification terms, with an optimum number as shown in Table. II and Table. III ( 5 for the Color database, and 3 or 5 for the Defect database). Notice that the over-fuzzification phenomenon also exists.

2) Influence of the fuzzification curves: In the Iris database case, the simplicity of the database doesn't allow to evaluate the influence of the fuzzification curves on the recognition rates. We observe the same conclusions for the Color database, because of the characteristic vector simplicity (3 attributes). That's why, we only present the results for the triangular case, which gives the "better" results (Table. II).

Contrary to the two previous cases, the complexity of the Defect database (9 parameters for the characteristic vector) allows to evaluate the influence of the fuzzification curves. The results presented in Table. III are the recognition rates obtained from four kinds of curves: trapezial curves, triangular curves, gaussian curves and trapezial-triangular curves. For all these kinds, we have evaluated the recognition rate for 6 decompositions: from 2 terms to 11 terms. In conclusion, we use for our industrial cases, the trapezial-triangular distribution with the 3 or 5 -terms split.

\section{TOWARDS AN INTERPRETABLE AND A MOST EFFICIENT NUMERIC MODEL: MODELLING AND INTEGRATING EXPERT KNOWLEDGE}

To take into account expert knowledge to build our numeric model comes from the following observations: to improve and to control a system we need the maximum of Information about this system. To access to this part of Information, we can use two sources: from human expert knowledge (expert in process, in products, in standards...)

TABLE III

RECOGNITION RATES ON DEFECTS SAMPLES IN GENERALIZATION

\begin{tabular}{ccccc}
\hline \hline $\begin{array}{c}\text { Numbers of } \\
\text { fuzzification } \\
\text { terms }\end{array}$ & $\begin{array}{c}\text { Trapezial } \\
\text { curves }\end{array}$ & $\begin{array}{c}\text { Triangular } \\
\text { curves }\end{array}$ & $\begin{array}{c}\text { Gaussian } \\
\text { curves }\end{array}$ & $\begin{array}{c}\text { Trapezial }+ \\
\text { Triangular } \\
\text { curves }\end{array}$ \\
\hline 2 & $43.6 \%$ & $19.0 \%$ & $41.0 \%$ & $46.7 \%$ \\
\hline $\mathbf{3}$ & $\mathbf{7 3 . 3 \%}$ & $\mathbf{7 8 . 5 \%}$ & $43.1 \%$ & $57.4 \%$ \\
\hline $\mathbf{5}$ & $70.3 \%$ & $76.9 \%$ & $\mathbf{7 4 . 9 \%}$ & $\mathbf{8 0 . 0} \%$ \\
\hline 7 & $69.7 \%$ & $75.4 \%$ & $71.3 \%$ & $70.8 \%$ \\
\hline 9 & $63.6 \%$ & $72.3 \%$ & $70.8 \%$ & $74.9 \%$ \\
\hline 11 & $59.5 \%$ & $66.2 \%$ & $70.3 \%$ & $68.2 \%$ \\
\hline \hline
\end{tabular}

who provides a rather qualitative piece of Information on the studied system; and from data acquisition directly trough the system, which gives a rather quantitative piece of Information provided in the form of measures. Linking these two sources as well different as complementary must contribute to lead to a more complete and coherent information modelling, allowing a stronger integration of the processes making up the system, and finally to contribute to a most efficient numeric model.

\section{A. General approach}

By referring to the literature, in the concerning field, systems which use human expertise and vision expertise, the main tendency resides in the building systems based on heuristic rules [14]-[16] which describe how the decisional system works. However, even if the set of rules seems to work, none precision is given on their validation compared to the initial knowledge given by the expert. They are thus only a-priori informal processes of validation. To avoid encountering this problem, we propose to use an approach based on a formal validation based on the N.I.A.M. method [17].

As the knowledge expressed by the expert is under the natural language form, the significant interest of this method resides in the fact that it relies directly on an expression of facts stated in natural language. The resulting information model could also be submitted to validation by the expert under a comprehensive form, i.e. in Binary Natural Language (BNL), which is a transcription of the information model also called "paraphrase action". The method allows completing progressively the model by asking to the expert precise questions induced by the method, also in natural language (for example: does a defect have one or several colors?). We thus obtain a model completed by totality and uniqueness constraints. The resulting models coming from the wood field end the vision field are called "symbolic" models.

The interest of this modelling and integration is to determine in a non-empiric way the more efficient parameters in input of each node of the FRC identification module in relation with the outputs. In other words, choose the optimal characteristic vector for each fuzzy inference engine. Fig. 4 shows our reflexion process.

The other interest of the modelling is to determine the number of fuzzification terms for each parameter. Instead of choosing empirically the number of term (for example, five equal terms), we can adapt this number of terms by considering expert knowledge. Reducing the number of fuzzification terms leads to simplify the numeric model (in term of number of rules generated).

Using the expert knowledge to determine the decisional structure of the numeric model and the number of fuzzification terms leads to a more interpretable numeric model. It allows to check it and validate it by comparing it with the initial knowledge used to build it. This part consists 


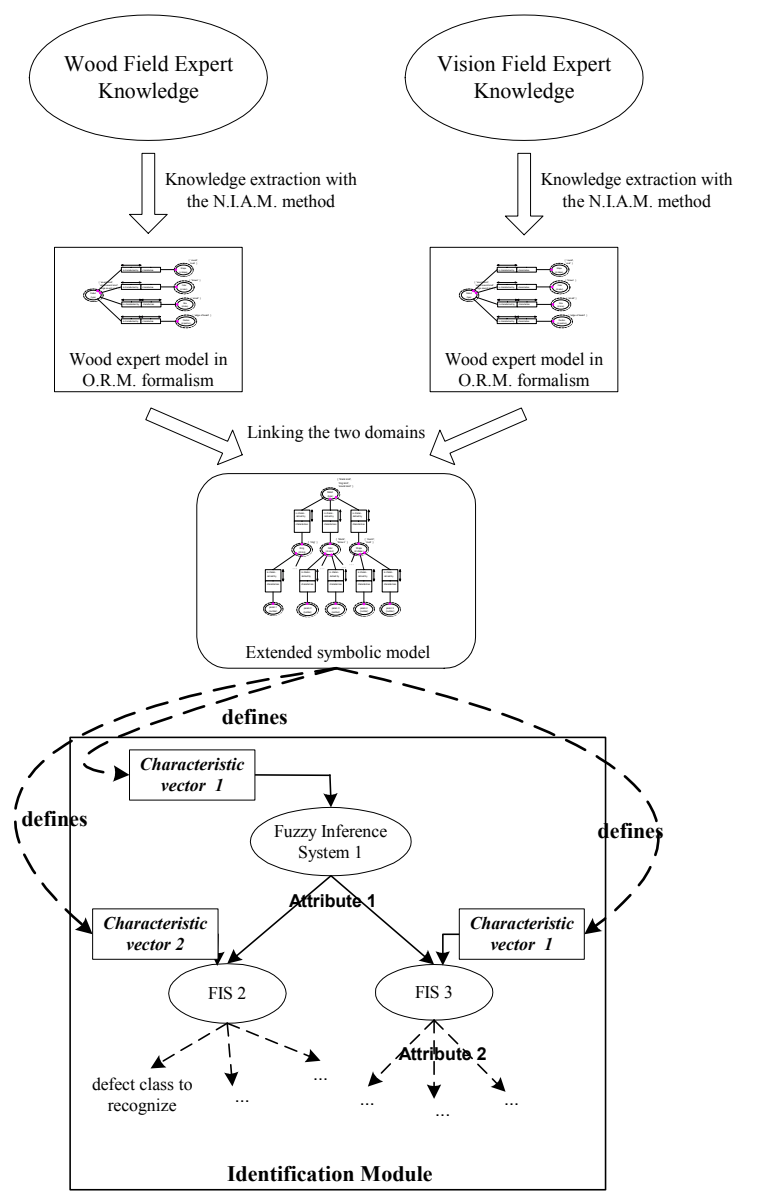

Fig. 4. Principle of the adopted method.

in analyzing the set of rules compounding the numeric model. To illustrate in a simple way our modelling and integrating thought process, we will only consider « sound knots family» defects type, that is to say sound knots, small sound knots and edge sound knots.

\section{B. Symbolic models}

We present in this section an overview of the N.I.A.M. method (Nijssen or Natural language Information Analysis Method) [17] and the O.R.M. (Object Role Modelling) formalism [18], [19] used to model the expert knowledge.

\section{1) N.I.A.M. method and O.R.M. formalism}

The NIAM method relies on a linguistic text analysis. From the expression of observable facts implicating objects, this method allows to distinguish non-lexical objects (NOLOTs) and lexical objects (LOTs) and the facts, which link them together. Fig. 5 illustrates some syntactical elements of the NIAM/ORM formalism allowing representing these objects and their relations.

For more information concerning the NIAM method and the proposed NIAM/ORM formalism, the reader can refer itself to [19].

2) Wood field and vision field symbolic models

To create this model, we ask the wood field expert to write a list in a natural language form, as complete as
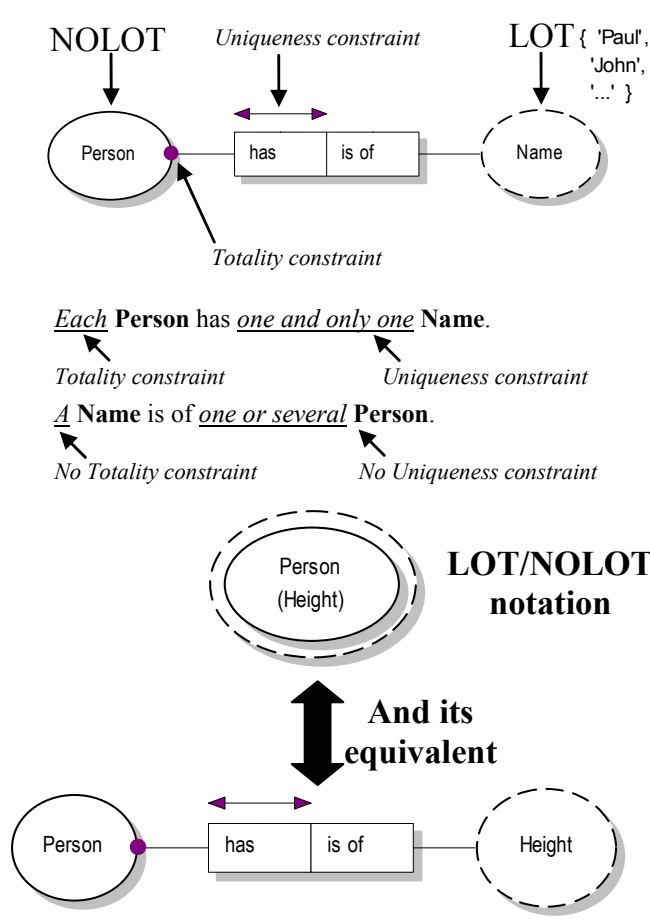

Fig. 5. The ORM formalism explained by an example.

possible, of the different defects that can be found in the species handled. This expression is sufficient to recognize in naked eye any kind of defect in a wood sample.

Fig. 6 represents the generic model for "knots" defect type including totality and uniqueness constraints characterizing a generic modelling.

In order to get this model validated by the expert who expressed the knowledge used for its development, we transcribe it in Binary Natural Language. The result of this transcription is thus a text that is closed to natural language, which does not impose to the expert any particular knowledge about the modelling method. Here is the text submitted to the expert for validation:

Each Defect is characterized by one and only one Shape. Each Shape characterizes one or several Defects. Each Defect is characterized by one and only one Color. Each

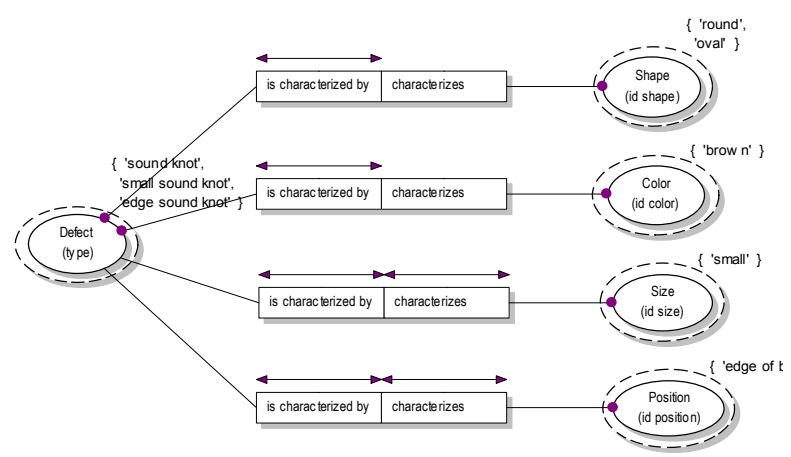

Fig. 6. Generic model of the sound knot "family".

Color characterizes one or several Defects. A Defect is 
characterized by one and only one Size. Each Size characterizes one and only one Defect. A Defect is characterized by one and only one Position. Each Position characterizes one and only one Defect.

The second model build concerns the field of expert vision. As done for "wood expert" symbolic model, we register the vision expert knowledge. This knowledge is about the defects identification system and more precisely on the parameters useful to quantify the characteristics of the defect to recognize. We apply the same principle than the one used for the wood expert symbolic model. We thus obtain a characterization of all the defects encountered in a vision parameter point of view.

Fig. 7 represents a partial formalization of the brown color concept with a vision point of view.

For instance, using this model, we are able to represent the following fact, by considering that a brown color is defined by other parameters than brown pixel number: a precise color is characterized by a unique brown pixel number. But the opposite is not true. To a given brown pixel number can correspond several brown colors.

We do the same for Shape, the Size and the Position characteristics. Notice that for confidential reasons we could not expose entirely the expert knowledge models. That is why just only some of the used parameters for each characteristic will be presented.

\section{Knowledge integration method}

The expert knowledge integration is done by linking expert knowledge from the two fields, i.e. wood field and vision field.

The process used to link these two domains consists in integrating the symbolic models. The resulting model is what we call an "extended" symbolic model. Fig. 8 illustrates a part of this model. For confidential reasons, we don't expose the vision parameters report to the different wood field concepts.

This model allows firstly to determine the most efficient parameters in input of each node compounding the numeric model and secondly to choose the most adapted fuzzification for each one of these parameters. Concerning the adapted fuzzification, let us consider for instance the Size wood concept and the reported vision parameters. For the wood expert, the Size can be small, medium or large. This leads to a 3-terms fuzzification for the parameters associated to the Size concept. Concerning the most efficient parameters to use in input of the nodes, the "extended" symbolic model allows to choose the best characteristic vector for each node.

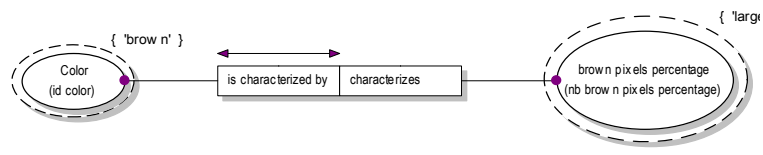

Fig. 7. Partial NIAM/ORM formalization for the brown color concept.

For the three examples of defects used to build the symbolic models (sound knot, small sound and edge sound knot), we can remark that the common characteristics are the Shape and the Color. Indeed, as the defects are all knots, their shape is thus similar and as they take part of the "sound knot

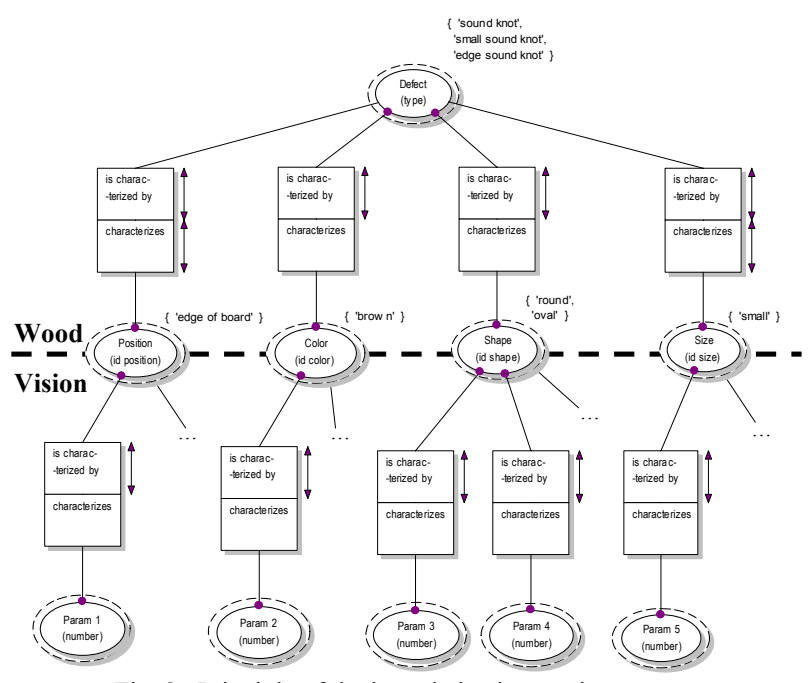

Fig. 8. Principle of the knowledge integration process.

family", their color is also similar. Thus only the Position and the Size characteristics are discriminating in their identification. The characteristic vector is thus compound only with the Size and Position vision parameters. Notice that the Color and the Shape concepts are used above in the tree structure of the model. They correspond to the two previous node of the structure, which allow to identify rounded defects from elongated defects and the different types of knot (ring, black and sound). Fig. 9 represents a part of the "arborescent" structure of the numeric model so built and more precisely the inference engine identifying the three defects taken as example.

\section{Numeric model interpretation}

The main interest of this integration by a Fuzzy Inference System based on a fuzzy linguistic rules system is the possibility of linguistically interpreting each inference of the model. It is thus possible to understand the numeric model behavior and check that expert knowledge was well integrated in the model and that there is coherence between

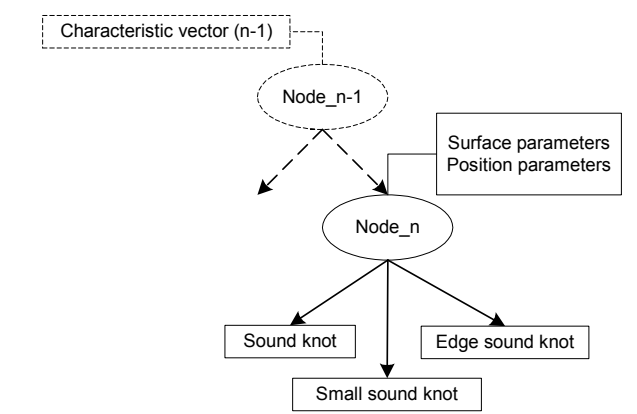

Fig. 9. Result of the knowledge integration into the numeric model constructing.

the input model data and the knowledge used to build it. A 
difference between data and knowledge can mean that used data are not really representative of the field or that expert knowledge initially expressed is neither sufficient nor complete.

This stage consists in interpreting the rule base obtained after the learning stage. To do that, we propose to use the matrix generated from the data sample in order to interpret the identification mechanism. This matrix is the numeric translation of fuzzy linguistic rules. It is composed as following:

- columns represent the parameters in input of the node,

- lines represent the rules.

To each premiss is associated a number which can be 0,1 or 2 in function of the number of fuzzification terms ("small", "medium", "large") used for the considered linguistic variable. To each fuzzy rule are associated a defect class (CS) and a confidence degree $(\mathrm{CF})$ conferred to the rule.

For instance, in the matrix generated for the node Node $n$ illustrated by Fig. 9, we obtain 16 fuzzy rules shown in Table. IV.

The matrix interpretation will be done with two aims. The first one is to improve the recognition rate of our method. For that, we propose to exclude the rules which are not discriminating for the defect or color identification. Different methods exist for that [9], [10]. We will use the CF confident coefficient calculated in the method and just exclude rules with $\mathrm{CF}$ coefficient strictly equal to zero. The second aim is to check if the components used to build the characteristic vector in relation with the knowledge are relevant. In the contrary case, the result of this part could be a questioning of knowledge models. An expert can have a subjective idea on the necessity of a parameter, which could be in reality not very relevant.

In Table. IV, we can remark for the rules 3 and 4 that the variation of the first parameter (parameter belonging to the Size concept) from the " 0 " value (corresponding to "small") to the " 1 " value (corresponding to "medium") does not influence the recognized defect class (defect class 2). By analyzing the matrix, on 16 rules, 13 underline the same behaviour (variation from " 0 " to " 1 ", or from " 1 " to " 2 "). We thus can wonder if this parameter is relevant to identify these three defects. To check it, we decide to create a new numeric model without the first parameter in input of this

TABLE IV

EXTRACT OF THE MATRIX GENERATED BY THE NODE N

\begin{tabular}{llllll}
\hline \hline Param_0 & Param_1 & Param_2 & Param_3 & CS & CF \\
\hline 0 & 0 & 0 & 0 & 1 & 0.427722 \\
1 & 0 & 0 & 0 & 0 & 0.730454 \\
0 & 1 & 0 & 1 & 2 & 1.000000 \\
1 & 0 & 0 & 1 & 2 & 1.000000 \\
$\ldots$ & $\ldots$ & $\ldots$ & $\ldots$ & $\ldots$ & $\ldots$ \\
\multicolumn{7}{l}{ Param_0 to Param 3 represents the premises } \\
CS the number of the defect class recognized \\
CF the confidence degree associated to the rule
\end{tabular}

TABLE V

COMPARED RECOGNITION RATES: KNOWLEDGE INTEGRATION VS NO KNOWLEDGE INTEGRATION

\begin{tabular}{lll}
\hline \hline \multicolumn{1}{c}{ Method } & \multicolumn{1}{c}{ Model } & $\begin{array}{c}\text { Recognition } \\
\text { rate }\end{array}$ \\
\hline Point method & 1: Statistic & $68.8 \%$ \\
Fuzzy method & 2: SI with equal distribution & $69.7 \%$ \\
& 3: AIS with equal distribution & $73.7 \%$ \\
& 4: SI with non-equal distribution & $77.4 \%$ \\
& 5: AIS with non-equal distribution & $83.6 \%$ \\
& 6: AIS interpreted with non-equal & $\mathbf{8 5 . 7 \%}$ \\
& distribution & \\
&
\end{tabular}

node. By deleting this parameter, instead of 16 rules, the resulting model is compound of 5 rules. The result obtained is given in Table. $\mathrm{V}$ (model 6).

The other interest of this interpretation is that we can refine the vision expert knowledge by deleting this parameter from the list of parameters characterizing the Size of a defect. This method is not yet automated but our purpose is to demonstrate the potentiality of the interpretation in obtaining a most efficient numeric model.

\section{E. Results}

We have tested three numeric models. The first one is directly derived from the LuxScan Technologies Company and is currently utilized by the company. The second one is the Single Inference (SI model) which does not take into account expert knowledge. The third one is the Arborescent Inference Structure (AIS model) with expert knowledge integration to take into account the industrial time processing constraint. And finally, the AIS model interpreted, i.e. the same model than the one used for the AIS model but without one of the Size parameter, which seems to be useless.

Moreover, we have tested two different types of fuzzification to analyze its influence on the recognition rate: an equal distributed fuzzification with an equal splitting of the representation space of each variable in 3 terms; a nonequal distributed fuzzification with a splitting in 2 or 3 terms according to expert knowledge.

Table. V summarizes the results obtained with two methods. These tests were led on the Defect database (see section 3.d.).

The main conclusions from these tests are the following. Firstly, in term of identification rate, the fuzzy method gives better results than the Point method. In all cases tested, the worst result given by the fuzzy method is better than the Point method.

Secondly, by only considering the results given for the different fuzzifications and the different models, we can see that the better result is given with an "interpreted" arborescent model with a representation space cutting, done in function of expert knowledge (what we call a non-equal distribution). This "interpreted" model is better in term of recognition rate, because deleting a non-discriminating parameter allows to delete ambiguous rules in the model; 
rules which lead to misclassifications. These results confirm our choice of using and integrating expert knowledge to build our numeric model and to specify the number of fuzzification terms for each parameter.

\section{CONCLUSION}

In this paper, we presented a Fuzzy Inference System based on fuzzy linguistic rules applied to industrial wood problems, i.e. wood color recognition and wood defects identification. Through this article, we shown the influence of the fuzzification curves and the number of fuzzification terms on the final recognition rate. Moreover, we show that integrating expert knowledge to enhance our decisionmaking system and interpreting the fuzzy rule base generated improves in a significant way the obtained results.

We notice that this Fuzzy Reasoning Classifier is currently implemented in the industrial software environment of the Company and is fully operational in a real wood production line.

Future works concern the improvement of the decisionmaking model from three points of view. Firstly, we want to improve the "arborescent" structure, currently built empirically, by integrating knowledge allowing to build the tree structure, i.e. allowing to define the link between the different inference engines compounding the model. This knowledge concerns hierarchy between defect attributes, that is to say the attributes order of importance to identify a defect. Secondly, we work to enhance the fuzzification step by defining an "automatic" fuzzification, i.e. a fuzzification which takes into account knowledge from the expert and data from the learning sample. Thirdly, we want to improve the expert knowledge modelling, and by the same, the expert knowledge integration by following the study on Fuzzy NIAM [20] initiated by M. Zgorzelski and Z. Zalewski. From the rough concepts expressed in this study, we want to refine them to propose a real applicable frame for this extension, and to apply it to our problem. Indeed, we have already introduced the notion of fuzzy values domain in our work but we want to go further by putting the imprecision and uncertainty notions in place in our extension.

\section{ACKNOWLEDGMENT}

The authors wish to thank their industrial partner the LuxScan Technologies Company, their staff and especially M. Raphaël Vogrig who is the general manager of the company and M. Jean-Yves Hergott who is responsible for projects development in the company and who follows the present works.

\section{REFERENCES}

[1] M. F. Kabir, D. L. Schmoldt, and M. E. Schafer, "Ultrasonic detection of knots, cross grain and bark pockets in wooden pallet parts", Proc. Of World Conference on Timber Engineering 2000, Whistler, British Columbia, Canada, Aug. 2000, pp. 7.5.2-1-7.5.2-8.

[2] H. Kauppinen, H. Rautio, and O. Silven, "Non-segmenting defect detection and SOM based classification for surface inspection using color vision", Conf. on Polarization and Color Techniques in Industrial Inspection (SPIE 3826), Munich, Germany, 1999, pp. 270280 .

[3] J. Lampinen, S. Smolander, and M. Korhonen, "Wood surface inspection system based on generic visual features", International Conference on Artificial Neural Networks ICANN'95, Paris, 1995.

[4] D.T. Pham, and S. Sagiroglu, "Training multilayered perceptrons for pattern recognition: a comparative study of four training algorithms", International Journal of Machine Tools \& Manufacture, vol. 41, 2001, pp. 419-430.

[5] L.A. Zadeh, "Fuzzy sets", Information and control, vol. 8, 1965, pp. 338-353.

[6] B. Bouchon-Meunier, "The fuzzy logic and its applications" (in French), Addison-Wesley, 1995.

[7] A. Kaufmann, "Introduction à la théorie des sous-ensembles flous", Edition Masson, 1975.

[8] D. Dubois, and H. Prade, "Fuzzy rules in knowledge-based systems Modelling gradedness, uncertainty and preference. An introduction to fuzzy logic application in intelligent systems", Kluwer, Dordrecht, 1992, pp. 45-68.

[9] L. Ughetto, "Fuzzy rule-based systems. Checking the consistency and inference methods" (in French), PhD thesis, INRIT, Toulouse, 1998.

[10] M.R. Berthold, "Mixed fuzzy rule formation", Int. Jour. of Approximate Reasoning, vol. 32, 2003, pp. 67-84.

[11] H. Ishibuchi, K. Nozaki, and H. Tanaka, "Distributed representation of fuzzy rules and its application to pattern classification", Fuzzy Sets and Systems, vol. 52, 1992, pp. 21-32.

[12] H Ishibuchi, K. Nozaki, N. Yamamoto, and H. Tanaka, "Construction of fuzzy classification systems with rectangular fuzzy rules using genetic algorithms", Fuzzy sets and systems, vol. 65, 1994, pp. 237253.

[13] H. Ishibuchi, K. Nozaki, and H. Tanaka, "A Simple but powerful heuristic method for generating fuzzy rules from numerical data", Fuzzy sets and systems, vol. 86, 1997, pp. 251-270.

[14] S.Y. Foo, "A rule-based machine vision for fire detection in aircraft dry bays and engine compartments", Knowledge-Based Systems, vol. 9 , no. 8, 1996, pp. 531-540.

[15] C.S. Wang, and X.K. Gong, "A fuzzy approximate reasoning model for a rule-based system in laser threat recognition", Fuzzy Sets and Systems, vol. 96, no. 2, 1998, pp. 139-146.

[16] Z. Wen, and Y. Tao, "Building a rule-based machine-vision system for defect inspection on apple sorting and packing lines", Expert Systems with Applications, vol. 16, 1999, pp. 307-313.

[17] H. Habrias, The binary relational model. I.A. method (NIAM) (in French), Eyrolles editions, 1998.

[18] H. Halpin, Information Modelling and Relational Databases, Morgan Kaufman edition, 2001.

[19] H. Halpin, Object Role Modelling (ORM/NIAM), Handbook on Architectures of Information Systems, 1998.

[20] M. Zgorzelski, and Z. Zalewski, "Fuzzy NIAM for real world data modeling", I.S.A.T.A. $29^{\text {th }}$ International Symposium on Automative Technology and Automation, Florence, Italy, 1996. 